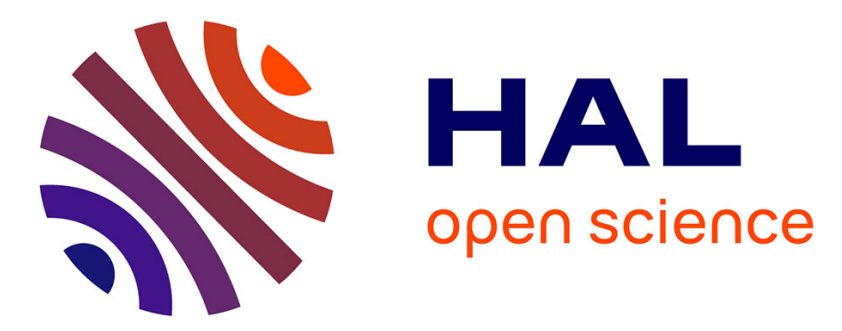

\title{
4D holographic reconstruction of embryonic blood flow by greedy algorithm
}

\author{
Alexey Brodoline, Dario Donnarumma, Daniel Alexandre, Michel Gross
}

\section{To cite this version:}

Alexey Brodoline, Dario Donnarumma, Daniel Alexandre, Michel Gross. 4D holographic reconstruction of embryonic blood flow by greedy algorithm. Digital Holography and Three-Dimensional Imaging 2016, Jul 2016, Heidelberg, Germany. pp.DTh1E.1, 10.1364/dh.2016.dth1e.1 · hal-01927511

\section{HAL Id: hal-01927511 https://hal.science/hal-01927511}

Submitted on 19 Nov 2018

HAL is a multi-disciplinary open access archive for the deposit and dissemination of scientific research documents, whether they are published or not. The documents may come from teaching and research institutions in France or abroad, or from public or private research centers.
L'archive ouverte pluridisciplinaire HAL, est destinée au dépôt et à la diffusion de documents scientifiques de niveau recherche, publiés ou non, émanant des établissements d'enseignement et de recherche français ou étrangers, des laboratoires publics ou privés. 


\title{
4D holographic reconstruction of embryonic blood flow by greedy algorithm
}

\author{
Alexey Brodoline, Dario Donnarumma, Daniel Alexandre and Michel Gross \\ Laboratoire Charles Coulomb - UMR 5221 CNRS-Université Montpellier, Place Eugène Bataillon, 34095 \\ Montpellier, France; \\ alexey.brodoline@umontpellier.fr
}

\begin{abstract}
An holographic microscopy setup using two illumination beams is proposed to image blood microcirculation in preclinical models. Greedy algorithm reconstruction of the two resulting holograms leads to a 3D reconstruction of the sample over time.

OCIS codes: (090.1995) Digital holography; (090.2880) Holographic interferometry; (170.3340) Laser Doppler velocimetry; (180.3170) Interference microscopy; (290.5850) Scattering, particles; (300.6310) Spectroscopy, heterodyne.
\end{abstract}

\section{Citation}

A. Brodoline, D. Donnarumma, D. Alexandre, and M. Gross, "4D holographic reconstruction of embryonic blood flow by greedy algorithm," in Imaging and Applied Optics 2016, OSA Technical Digest (online) (Optical Society of America, 2016), paper DTh1E.1. DOI: 10.1364/DH.2016.DTh1E.1

Conference Digital Holography and Three-Dimensional Imaging 2016 Heidelberg Germany 25-28 July 2016 From the session Contemporary Topics in DH (DTh1E) ISBN: 978-1-943580-15-6

\section{Introduction}

Microvessel blood flow imaging techniques are widely used in biomedical research and clinical diagnostics where many diseases have a vascular etiology or involvement. However, most of the existing techniques are invasive due to the use of a contrast agent for imaging purposes. Recent developments in Digital Holography and Laser Doppler Holography techniques can be considered to alleviate this issue. [1]. Laser Doppler holography and transmission microscopy can be coupled [2] to analyze blood flow in fish embryos by adapting a laser Doppler holographic setup to a standard bio-microscope [3].

Two illumination beams oriented in two different directions have been used to enhance the slicing along $z$. Thus, each illumination beam signal has been selected with the aim to obtain two holograms from which two 3D intensity maps have been calculated. The highest values of correlation of these intensity maps have been selected by using a greedy algorithm to obtain a 3D reconstructed image. This procedure has been repeated over time to obtain a $4 \mathrm{D}$ reconstruction of the moving objects. The technique has been validated by imaging of fish embryos blood microcirculation.

\section{Experimental setup and methods}

The presented setup (see Fig. 1 (a)) consists in an upright microscope that has been modified for making heterodyne holography [4]. The main laser (frequency $\omega_{I}$ ) is split by a beam splitter into two arms (illumination and reference). Two acousto optic modulators (AOM) at $\omega_{1,2} \simeq 80 \mathrm{MHz}$ are able to control the frequency $\omega_{L O}=\omega_{I}+\omega_{1}-\omega_{2}$ of the reference $\operatorname{arm}\left(E_{L O}\right)$. The illumination $\operatorname{arm}(E)$ has been split again into two beams so as to illuminate the sample from two different directions. The imaged sample is a zebrafish embryo (48 hpf) between a slide and a cover slip. Both illumination beams pass through the sample and enter inside a microscope objective $\mathrm{MO}(\mathrm{NA}=0.30, \mathrm{G}=10)$, as shown in Fig. 1 (b). The beam splitter that recombines the signal $(E)$ and reference $\left(E_{L O}\right)$ fields is angularly tilted to achieve off-axis holography: $\theta \neq 0$. The CCD camera $\left(1280 \times 1024\right.$ pixels, $\omega_{C C D}=220 \mathrm{~Hz}, 10$ bits $)$ records the interference pattern $I=\left|E+E_{L O}\right|^{2}$. Sequences of 256 frames are recorded: $I_{0} \ldots I_{255}$.

Recorded data are cropped into a $1024 \times 1024$ calculation grid to perform holographic calculations like Fast Fourier Transforms (FFT). To select the signal corresponding to each illumination, we have reconstructed the image of the field $E$ scattered by the sample in the MO back pupil plane. Reconstruction is made with 4 phases holograms $H=$ $\left(I_{0}-I_{2}\right)+j\left(I_{1}-I_{3}\right)$, and with $\omega_{1}-\omega_{2}=\omega_{C C D} / 4$. Images are displayed in intensity by averaging data over 80 frames. 


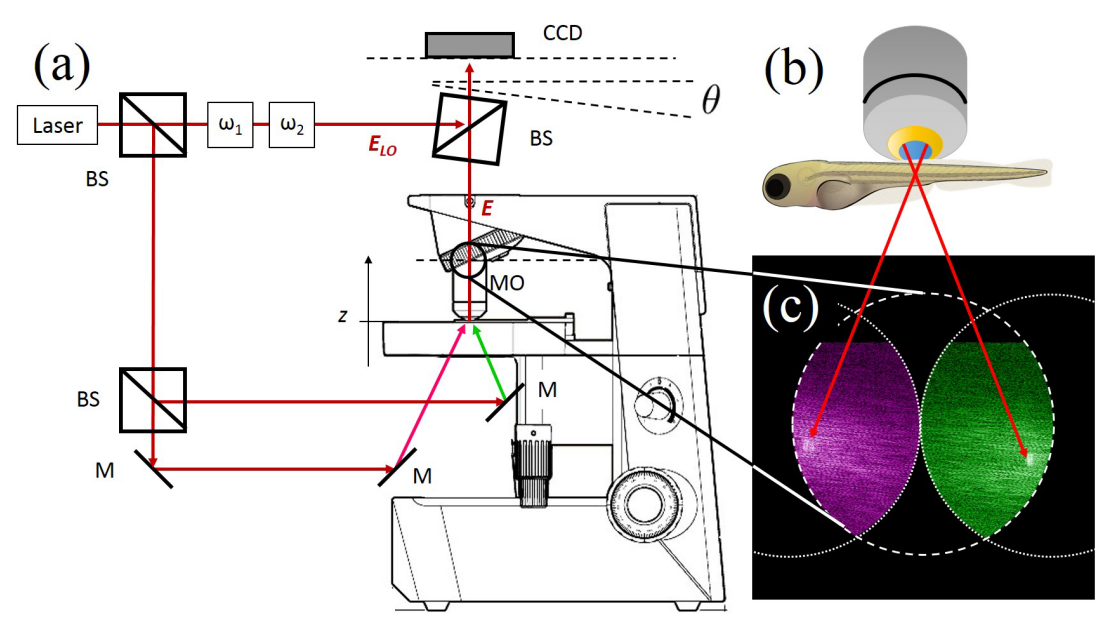

Fig. 1. a) Holographic microscopy setup: BS: beam splitter; M: mirror; $\omega_{1}, \omega_{2}$ : Acousto optic modulators (Bragg cells) that shift the frequency of the local oscillator beam. MO: microscope objective; CCD: CCD camera; $E$ and $E_{L O}$ : signal and reference complex fields. b) Two illumination beams go through the embryos and enter in the high numerical aperture objective. c) Pupil images reconstructed with 4 phases holograms $H=\left(I_{0}-I_{2}\right)+j\left(I_{1}-I_{3}\right)$, and with $\omega_{1}-\omega_{2}=\omega_{C C D} / 4$ for a zebrafish sample; the two beams are selected with different colors.

The pupil image, which is located in the upper right hand size of the calculation grid, has been displayed in the center of Fig. 1 (c). We have selected the useful holographic information by cropping the pupil zone (and by filling the rest of the calculation grid with zeros). The two illumination beams yield two bright spots due to the transparency of the zebrafish sample. Since these two spots are well separated in the Fourier space, is it possible to separate each contribution by cropping 2 circular zones of radius 240 pixels centered on each spot (dotted white circle of Fig. 1 (c) ) in order to perform reconstruction with one of the two illumination beams (i.e with the purple and green zones of Fig. $1(\mathrm{c}))$.

\section{3D reconstruction by greedy algorithm}

In previous work we have exploited the $z$ information of the coincidence of purple and green images obtained with double illumination beams $[3,5,6]$.

In this work we have made the 2 beams reconstruction by considering 2 phases holograms $H_{2 \varphi, n}=I_{n}-I_{n+1}$ with $\omega_{1}-\omega_{2}=0$ so to image the moving RBCs (and thus the blood vessels by averaging the reconstructed images).

Thus, we have determined the 3D locations of the red blood cells (RBCs), by assuming that their $x, y, z$ locations correspond to coincidences in the 3D intensity maps $\left|H_{\text {purple }, n}(x, y, z)\right|^{2}$ and $\left|H_{\text {green }, n}(x, y, z)\right|^{2}$. Note that we have considered here coincidences in the scattered field intensities, because the phase of the scattered fields strongly depends on the illumination direction.

To be more precise, we have considered that the fields $H_{\text {purple }}(x, y, z)$ and $H_{\text {green }}(x, y, z)$ that are measured, result from $M$ individual complex sources $S_{\text {purple, } m}$ and $S_{\text {green, } m}$ with $m=1 \ldots . M$ and $M=25000$, located in points $x_{m}, y_{m}, z_{m}$. The sample is thus represented by the 3D complex maps of sources $S_{\text {purple, } m}(x, y, z)$ and $S_{\text {green }, m}(x, y, z)$ that represent the individual scatterers (i.e. the RBCs). In these 3D maps, only $M=25000$ locations are non zero. To perform the calculations, we have considered a calculation grid of $512 \times 512 \times 101$ points in $x, y$ and $z$ with a pitch of $1.138 \mu \mathrm{m}$ in $x$ and $y$ and $2 \mu \mathrm{m}$ in $z$.

We have started the calculation with $S_{\text {purple }}(x, y, z)=0$ and $S_{\text {green }}(x, y, z)=0$ for all points of the grid. We have then calculated the location of the first source point $(m=1)$. This was done by calculating $\left|H_{\text {purple }}(x, y, z)\right|^{2}$ and $\left|H_{\text {green }}(x, y, z)\right|^{2}$ and by assuming that the maximum correlation point $x_{1}, y_{1}, z_{1}$ corresponds thus to the maximum of the product $\left|H_{\text {purple }}(x, y, z)\right| \times\left|H_{\text {green }}(x, y, z)\right|$. We have considered that the purple and green first source points are $S_{\text {purple }}\left(x_{1}, y_{1}, z_{1}\right)=H_{\text {purple }}\left(x_{1}, y_{1}, z_{1}\right)$ and $S_{\text {green }}\left(x_{1}, y_{1}, z_{1}\right)=H_{\text {green }}\left(x_{1}, y_{1}, z_{1}\right)$. 


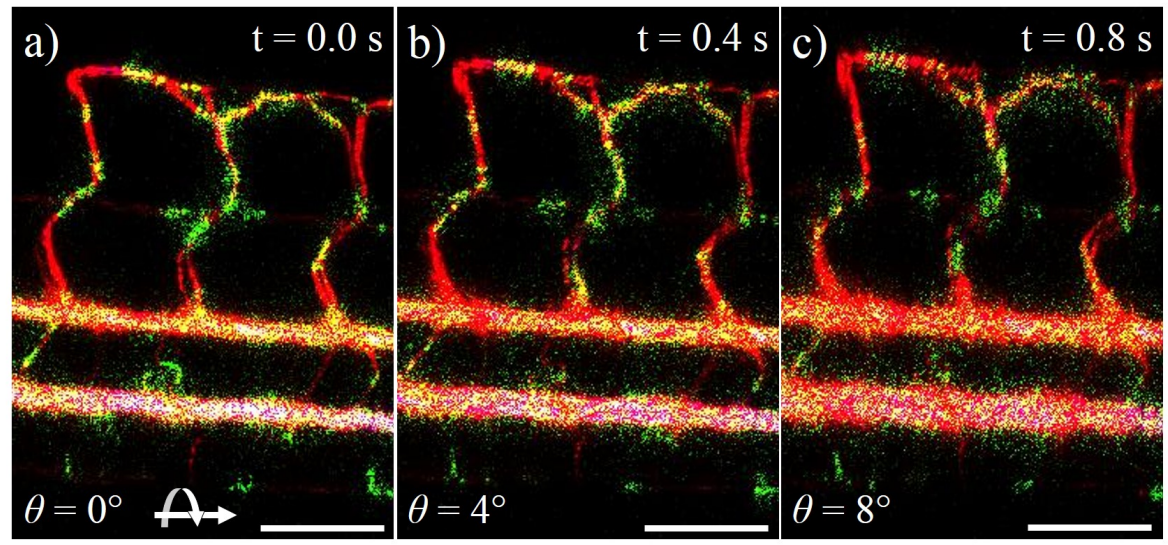

Fig. 2. 3D intensity images of vessels (red) and RBCs (green) flowing in time, reconstructed by greedy algorithm. This reconstruction has been done by calculating purple and green 3D maps $S_{\text {purple }}(x, y, z)$ and $S_{\text {green }}(x, y, z)$, and by assuming that the largest coincidence corresponds to the non-zero point. The displayed images (a..c) report the $3 \mathrm{D}$ reconstruction tilted by different angles and at different instants of time. Dimensional bar $=100 \mu \mathrm{m}$.

To calculate the second source point $(m=2)$, we have used a greedy algorithm. We have thus modified the holograms $H_{\text {purple }}\left(x, y, z_{1}\right)$ and $H_{\text {green }}\left(x, y, z_{1}\right)$ in plane $z=z_{1}$ that contains all the scattered light information by forcing to zero the point $x_{1}, y_{1}, z_{1}$ (since this point is already within $S_{\text {purple }}$ and $S_{\text {green }}$ ). We have then recalculated $\left|H_{\text {purple }}(x, y, z)\right|^{2}$ and $\left|H_{\text {green }}(x, y, z)\right|^{2}$ in all points of the grid with the modified $z=z_{1}$ plane hologram and we have calculate the location $x_{2}, y_{2}, z_{2}$ of the second maximum of $\left|H_{\text {purple }}(x, y, z)\right| \times\left|H_{\text {green }}(x, y, z)\right|$.

We have redo the same calculation for $3^{\text {th }}, 4^{\text {th }} \ldots m^{\text {th }}$ source points, and we got by this way the 3D maps $S_{\text {purple }}(x, y, z)$ and $S_{\text {green }}(x, y, z)$. This calculation has been done for all holograms $H_{2 \varphi, n}$ of the time sequence. The calculation is made with a GPU (NVidia GTX TITAN). For each time point $n$, the calculation of the $M=25000$ points of $S_{\text {purple }}$ and $S_{\text {green }}$ is made in about 20 minutes.

Once $S_{\text {purple }, n}(x, y, z)$ and $S_{\text {green }, n}(x, y, z)$ have been calculated for each time step $n$, we have calculated the time averages $\left\langle\left|S_{\text {purple }}\right|^{2}\right\rangle$ and $\left\langle\left|S_{\text {green }}\right|^{2}\right\rangle$. This average calculation has been made to better image the blood vessels.

The final result is a 3D image as in Fig. 2 (a...c), where both the RBCs (in green) and the vessels (in red) are shown at different time instants and with different rotation angles around the $y$ axe parallel to the large vessels.

\section{Conclusion}

In this paper, we proposed a technique that combines digital holography, dual illumination of the sample and reconstruction with a greedy algorithm to obtain 4D images of blood microcirculation in a living zebrafish embryo. Other quantitative applications of this technique [7] like the evaluation of the individual RBC motion with possible coincidence of images can be also studied from sequences of reconstructed images similar to the one displayed on Fig. 2.

All animal experiments that are described in the present study were conducted at the University of Montpellier according to European Union guidelines for the handling of laboratory animals and were approved by the Direction Sanitaire et Vétérinaire de l'Hérault and Comité d'Ethique pour l'Expérimentation Animale under reference CEEALR-13007.

Acknowledgments. We acknowledge Labex Numev (convention ANR-10-LABX-20) n. 2014-1-042 grant for funding.

\section{References}

1. O. Sakurada, C. Kennedy, J. Jehle, J. Brown, G. L. Carbin, and L. Sokoloff, "Measurement of local cerebral blood flow with iodo [14c] antipyrine," American Journal of Physiology-Heart and Circulatory Physiology 234, 
H59-H66 (1978).

2. M. Atlan, M. Gross, B. C. Forget, T. Vitalis, A. Rancillac, and A. K. Dunn, "Frequency-domain wide-field laser doppler in vivo imaging," Optics letters 31, 2762-2764 (2006).

3. N. Verrier, D. Alexandre, and M. Gross, "Laser doppler holographic microscopy in transmission: application to fish embryo imaging," Optics express 22, 9368-9379 (2014).

4. F. Le Clerc, L. Collot, and M. Gross, "Numerical heterodyne holography with two-dimensional photodetector arrays," Optics letters 25, 716-718 (2000).

5. D. Alexandre, G. Lutfalla, and M. Gross, "Holographic imaging of zebrafish embryo blood flow with dually oriented illumination beams," in "Digital Holography and Three-Dimensional Imaging," (Optical Society of America, 2015), pp. DTh2A-6.

6. M. Gross, N. Verrier, and P. Picart, "Fish embryo multimodal imaging by laser doppler digital holography," in "Signal Recovery and Synthesis," (Optical Society of America, 2014), pp. JTu4A-7.

7. D. Donnarumma, A. Brodoline, D. Alexandre, and M. Gross, "Blood flow imaging in zebrafish by laser doppler digital holography," (2016). Manuscript submitted for publication. 